\title{
Theoretical estimates of the convective turnover time for low-mass, rotating pre-main sequence stars
}

\author{
L. T. S. Mendes ${ }^{1,2}$, N. R. Landin ${ }^{2}$ and L. P. R. Vaz \\ ${ }^{1}$ Depto. de Engenharia Eletrônica, UFMG, C.P. 702, 31270-901 B. Horizonte, MG, Brazil \\ email: luizt@cpdee.ufmg.br \\ ${ }^{2}$ Depto. de Física, UFMG, C.P. 702, 31270-901 Belo Horizonte, MG, Brazil \\ email: nlandin@fisica.ufmg.br, vaz@fisica.ufmg.br
}

\begin{abstract}
The Rossby number $R o$ is an important quantity related to the well-known magnetic activity-rotation correlation for main sequence, solar-type stars. For such stars, Ro can be obtained by a semi-empirical relationship between the convective turnover time $\tau_{\mathrm{c}}$ and the $\mathrm{B}-\mathrm{V}$ colour index, but an equivalent activity-rotation correlation seems not to exist for pre-main sequence stars. In this work we report theoretical estimates of $\tau_{\mathrm{c}}$ for low-mass, rotating premain sequence stars under either the Full Spectrum of Turbulence (FST) or the classical Mixing Length Theory (MLT) convection models. The results for the MLT models show that the lower the convection efficiency the higher $\tau_{\mathrm{c}}$, while the FST models give $\tau_{\mathrm{c}}$ lower than those for the MLT. The presence of a parametric magnetic field lowers the convection efficiency, resulting in smaller $\tau_{\mathrm{c}}$ values.
\end{abstract}

Keywords. Stars : convection, stars: evolution, stars: rotation

\section{Introduction}

It is well known that the chomospheric and coronal emission in late-type stars, observed in optical, UV and X-ray wavelengths, are well correlated with stellar rotation. It is believed that such emission is powered by a magnetic field which, in turn, is generated by the interaction between rotation and convective motions at the star's outer envelope.

The activity-rotation correlation is usually better described in terms of the Rossby number Ro, defined as the ratio of the rotational period $P_{\text {rot }}$ to the convective turnover time $\tau_{\mathrm{c}}$. For main sequence stars, $\tau_{\mathrm{c}}$ is usually obtained as a function of the B-V colour index originally derived by Noyes et al. (1984) for their star sample.

In the case of pre-main sequence stars, X-ray observations have been used to probe their magnetic activity. However, recent studies (e.g. Preibisch et al. 2005) were not able to found a clear correlation between X-ray emission and rotation. Since there is no empirical function that gives $\tau_{\mathrm{c}}$ for pre-main sequence stars, theoretical models are the only way to support those studies. Here we report theoretical values of $\tau_{\mathrm{c}}$ obtained for rotating, pre-main sequence stellar models, and its dependencies on the convection model and on the presence of magnetic fields.

\section{The Models}

Rotating stellar models from $1.2 \mathrm{M}_{\odot}$ down to $0.2 \mathrm{M}_{\odot}$ were computed with the ATON 2.3 rotating evolutionary code (Ventura et al. 1998, Mendes et al. 1999). All models were computed with solar chemical composition, and under either the FST (Full Scale of Turbulence; Canuto et al. 1996) or the MLT (Mixing Length Theory) convection 

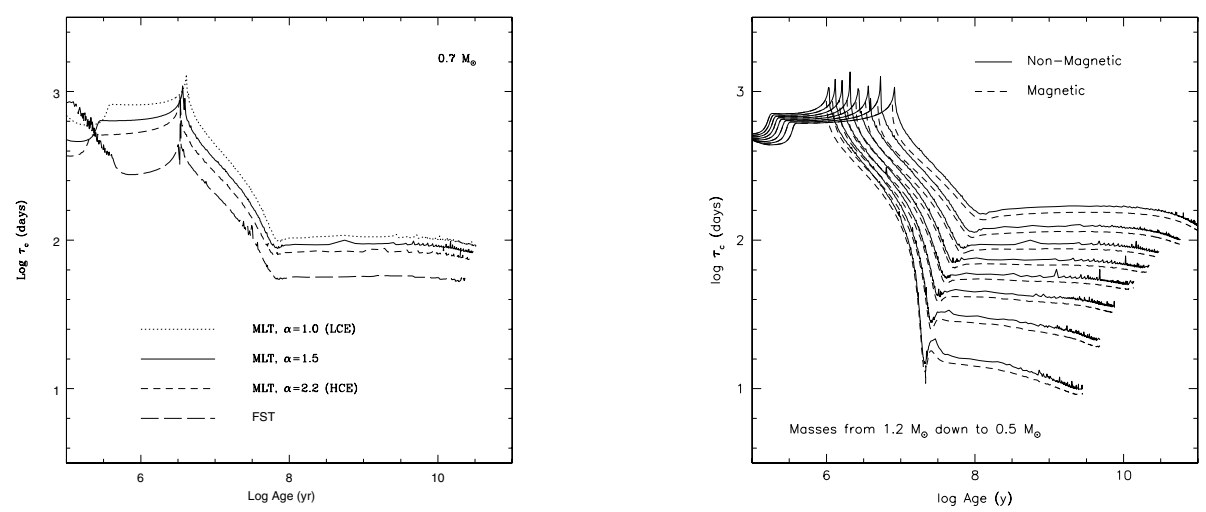

Figure 1. Left: Global convective turnover time as function of age for the $0.7 \mathrm{M}_{\odot}$ model, at different convective regimes. Right: Global convective turnover time as function of age for MLT models with $\alpha=1.5$, and considering the effects of a parametric magnetic field.

treatments; in the latter case, the models were run under values of $\alpha=\lambda / H_{\mathrm{p}}$ of 1.0 ("Low Convection Efficiency", or LCE), 1.5 (typical for a solar match for the $1.0 \mathrm{M}_{\odot}$ model) and 2.2 ("High Convection Efficiency", or HCE). The initial rotation value of the models were obtained from Kawaler (1987) relations between mass and angular momentum. This choice, besides being not arbitrary, results in the typical range of rotational periods observed for low-mass, pre-main sequence stars in star-forming regions.

\section{Results}

The results obtained for the rotating, pre-main sequence models show the same behaviour obtained elsewhere for non-rotating, main sequence stars (e.g. Pizzolato et al. 2001) regarding the chosen convection model: for MLT models, the higher the convection efficiency (larger $\alpha$ parameter) the lower $\tau_{c}$ and hence a higher Rossby number, as showed in the left side of Fig. 1. However, for masses above $1 \mathrm{M}_{\odot}$, we found that such trend is inverted as the star enters the main sequence. The FST models show in general lower values of $\tau_{c}$ than those for the MLT, as it describes the whole spectrum of convective eddies and, as so, convective velocities are computed in a intrinsically different way.

Since most low-mass, pre-main sequence stars show signatures of a strong magnetic field, a further test was to check the impact of the magnetic field on the convective turnover times. For that, we adopted the modified Schwarzchild criterium for convective instability first proposed by Gough \& Tyler (1966), namely

$$
\nabla_{\mathrm{rad}} \geqslant \nabla_{\mathrm{ad}}+\delta, \quad \delta=\frac{B^{2}}{B^{2}+\gamma P}
$$

and adopting the fixed value of $\delta=0.05$ for the whole star. The right side of Fig. 1 shows that the convective turnover times, in this case, are shorter than those for the usual non-magnetic models.

\section{Application to the ONC Stars}

The ATON 2.3 code has recently been used for a study of evolutionary and rotational properties of low-mass stars in the Orion Nebula Cloud (ONC), in which new sets of premain sequence evolutionary tracks including rotation and non-grey atmospheres were 

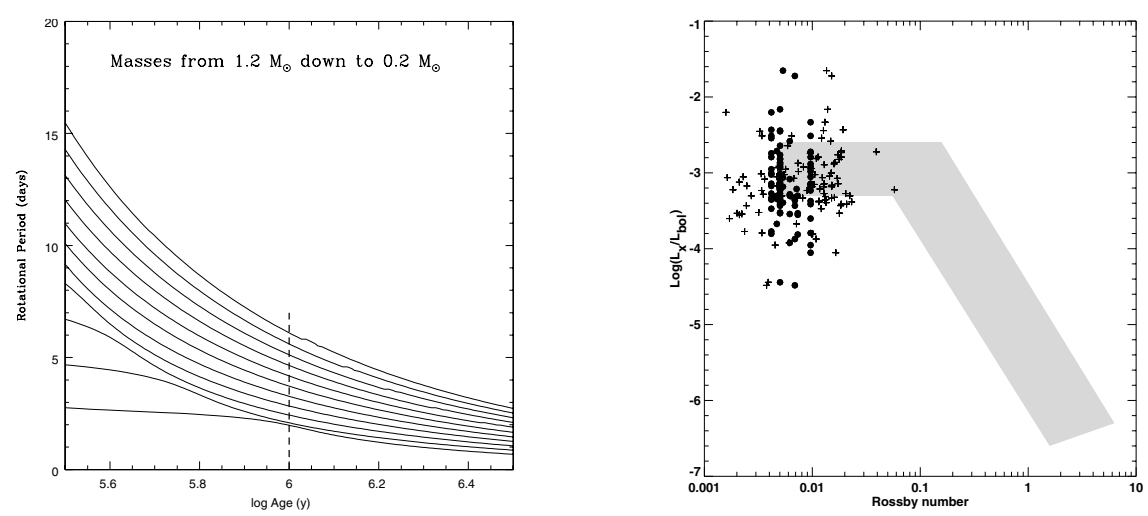

Figure 2. Left: Rotational periods for the rotating pre-main sequence models. All models were started with an initial angular momentum according to Kawaler's (1987) prescriptions. The dashed vertical line highlights the model periods at the ONC age. Right: X-ray luminosties of the ONC pre-main sequence stars as a function of the Rossby number. The ONC data is from Stassun et al. (2004). The filled circles indicate Rossby number values computed by using the theoretical values of both $\tau_{c}$ and the rotation period. The gray shaded region shows the main-sequence relationship (Pizzolato et al. 2003) for comparison.

generated (Landin et al. 2006). One of the main results of that study is the confirmation of a double-peaked distribution of rotational periods for stars with masses lower and higher than $0.5 \mathrm{M}_{\odot}$.

As an application of the theoretical values of $\tau_{c}$ computed for rotating pre-main sequence models, we computed the Rossby numbers for the ONC star sample, adopting either the observed rotation periods or those ones from the rotating models at the age of the ONC $\left(10^{6}\right.$ years). Figure 2 on its left side shows that the theoretical rotation periods of the models cover the observed period range, while its right side confirms that the X-ray luminosities of the ONC pre-main sequence stars do not shown a strong correlation with the Rossby number as observed for main-sequence stars.

\section{Acknowledgements}

The authors thank Drs. Italo Mazzitelli and Francesca D'Antona (INAF, Italy) for allowing them full access to their evolutionary code ATON 2.0. L.T.S. Mendes acknowledges the partial support of IAU and of the Czech Academy of Sciences for attending this meeting.

\section{References}

Canuto, V.M., Goldman, I., \& Mazzitelli, I. 1996, ApJ 473, 550

Gough, D.O. \& Tayler, R.J. 1966, MNRAS 133, 85

Kawaler, S.D. 1987, PASP 99, 1322

Landin, N., Ventura, P., D’Antona, F., Mendes, L.T.S., \& Vaz, L.P.R. 2006, A\&A 456, 269

Mendes, L.T.S., D'Antona, F., \& Mazzitelli, I. 1999, A\&A 341, 174

Noyes, R.W., Hartmann, L.W., Baliunas, S.L., Duncan, D.K., \& Vaughan, A.H. 1984, ApJ 279,763

Pizzolato, N., Ventura, P., D’Antona, F., Maggio, A., Micela, G., \& Sciortino, S. 2001, A\&A 373,597

Pizzolato, N., Maggio, A., Micela, G., Sciortino, S., \& Ventura, P. 2003, A\&A 397, 143

Preibisch, T., Kim, Y.-C., Favata, F., Feigelson, E.D., Flaccomio, E., Getman, K., Micela, G., Sciortino, S., Stassun, K., Stelzer, B., \& Zinnecker, H. 2005, ApJS 160, 401

Stassun, K.G., Ardila, D.R., Basri, G., \& Mathieu, R.D. 2004, AJ 127, 3537

Ventura, P., Zeppieri, A., Mazzitelli, I., \& D'Antona, F. 1998, A\&A 334, 953 